\title{
Fulminant Neurologic Manifestation of Systemic Lupus Erythematosus
}

\author{
Catalina Bistriceanu ${ }^{1, *}$, Florentina Anca Danciu, Lucian Cracana ${ }^{1}$, Elena Rezus², Carmen \\ Volovat $^{3}$
}

1 "Prof. Dr. N. Oblu" Neurosurgery Clinical Emergency Hospital, lasi, Romania, ${ }^{2}$ Rheumatology Department, Rehabilitation Hospital lasi, "Grigore T. Popa" University of Medicine and Pharmacy, lasi, Romania, ${ }^{3}$ Nephrology Department, University Hospital "Dr. C. I. Parhon", Iasi, Romania

\begin{abstract}
Central nervous system (CNS) presentations of Systemic Lupus Erythematosus (SLE) may be focal neurological and diffuse psychiatric manifestations. We are reporting a clinical case of severe neurologic manifestations on a 19 year old woman with SLE diagnosed in the Neurology clinic. Severe neurological involvement in SLE is one of the most dreadful complications of the disease, associated with a poor prognosis. The problem remains what is the molecular support of such an important cerebral event. Blood brain barrier endothelium is apparently a target for several pathogenic mechanisms that can account for the CNS manifestations of SLE. The autoimmune response in SLE might affect endothelial cells through several mechanisms and different mediators: endothelial activation/damage may be mediated by $\mathrm{C} 1 \mathrm{q}$.
\end{abstract}

Keywords: Systemic Lupus Erythematosus, central nervous system, blood brain barrier, necrotizing encephalitis

\section{Introduction}

Systemic lupus erythematosus (SLE) is a systemic autoimmune disease characterized by hyper-activation of $B$ and $T$ lymphocytes resulting in the overproduction of autoantibodies, tissue deposition of immune complexes, and high levels of inflammatory cytokines, cumulatively resulting in a systemic pro-inflammatory state [1].

Central nervous system (CNS) presentations in SLE patients consist of a broad array of symptoms, which can be generally divided between focal neurological and diffuse psychiatric manifestations. Focal episodes may include seizures and cerebrovascular events, while psychiatric presentations often consist of anxiety and depression [2].

Received: October 2014; Accepted after review: November 2014; Published: December 2014

${ }^{*}$ Corresponding author: Catalina Bistriceanu, MD, "Prof. Dr. N. Oblu" Neurosurgery Clinical Emergency Hospital, 1 Ateneului Str., lasi, Romania.

Email: catalina nastac@yahoo.com
Neuropsychiatric SLE is often associated with the presence of neuropathic antibodies within the CNS, making the question of how they gain entry into this anatomically privileged space increasingly important.

There is strong support for the roles of anti-endothelial cell antibodies, complement components, and environmental mediators in increasing permeability across the blood brain barrier (BBB), though in each of these cases, cytokines and chemokines have an essential role as well [3].

\section{Case report}

A 19 year-old female is admitted in a local Neurologic Clinic for the acute onset of complete motor deficit of her left limbs, swallowing disorders and speech problems. The symptoms were preceded by general state alteration and swallowing pains 2-3 days prior to her admittance. 
No autoimmune disease was found in her family history and no evidence of drugs or other toxic substances was found. Six months ago the patient was diagnosed with pernio erythema (cutaneous distal lesions of her upper and lower limbs with erythema, pain and petechiae at the distal phalanges worsened in cold exposure).

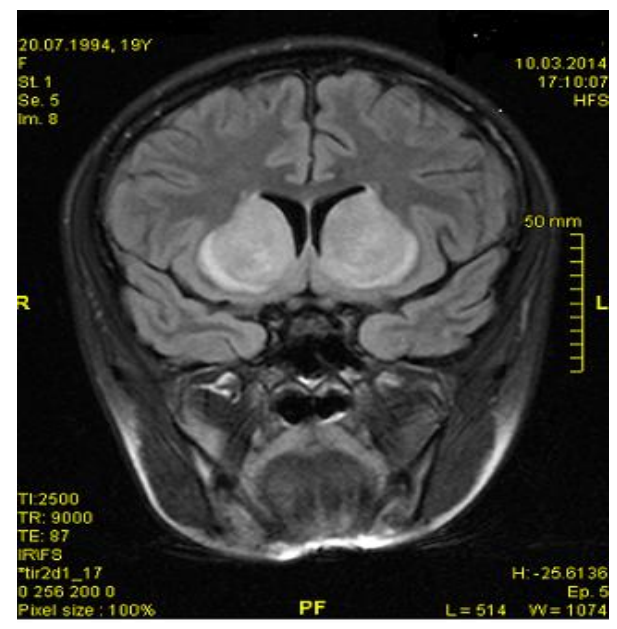

Fig. 1. Brain RM imaging (T2 weighted dark fluid) revealed bilateral symmetrical high signal intensities in both thalami on coronal section

The lumbar puncture yielded 0 cells and twice the normal level of proteins in the CSF exam. Immunologic investigations were done, including the ANAs extensive profile, the anticardiolipin immunoglobulin $\mathrm{G}[\mathrm{Ig} \mathrm{G}]$ and $\mathrm{M}$ [ $\mathrm{Ig}$ $\mathrm{M}]$, lupic cells which were absent, the HVC antibodies - negative, the $\lg \mathrm{CMV}$ and toxoplasma - negative, HIV - negative, HBS negative and VDRL - negative. Blood analysis showed pancytopenia and hepatic cytolysis.

The patient was transferred into our clinic (Neurology Clinic, "Prof Dr. N. Oblu" Neurosurgery Emergency Hospital, lasi). Clinical examination on admittance revealed: remitted spontaneous anterior epistaxis, nasopharyngeal mucous secretions, oral mucosae with bleeding on oral examination, "butterfly" facial erythema (Figure 3), petechial eruption and trophic disorders at distal phalanges - upper and lower limbs, erythematous plaque of thighs, sinus bradycardia - 45 BPM (probable due to the central bulbar involvement) responsive to atropine, mild pulmonary involvement: polipnea and increased vesicular murmur.
In the clinic where she was first admitted the MRI showed the encephalitis aspect consisting of vasogenic edema with hemorrhagic petechiae at the bilateral thalamus (Figure 1) and brainstem (Figure 2).

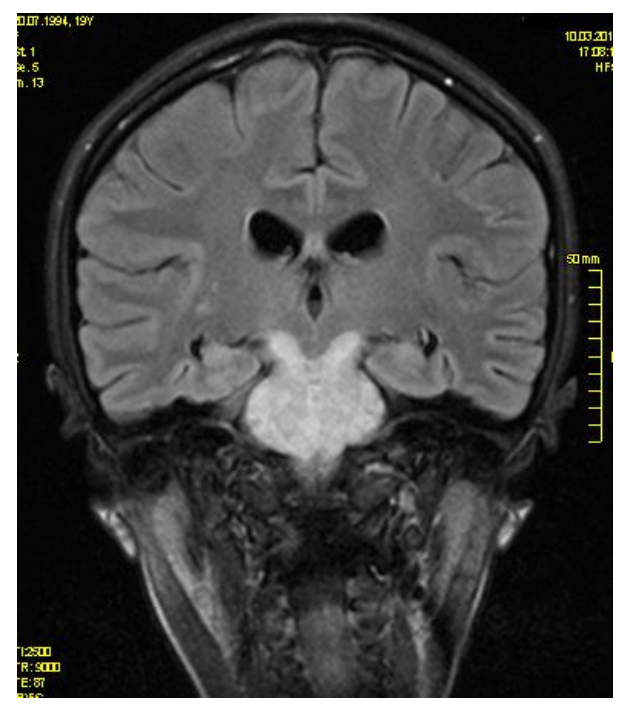

Fig. 2. Brain $R M$ imaging (T2 weighted dark fluid) revealed high signal intensity of the brainstem on coronal section

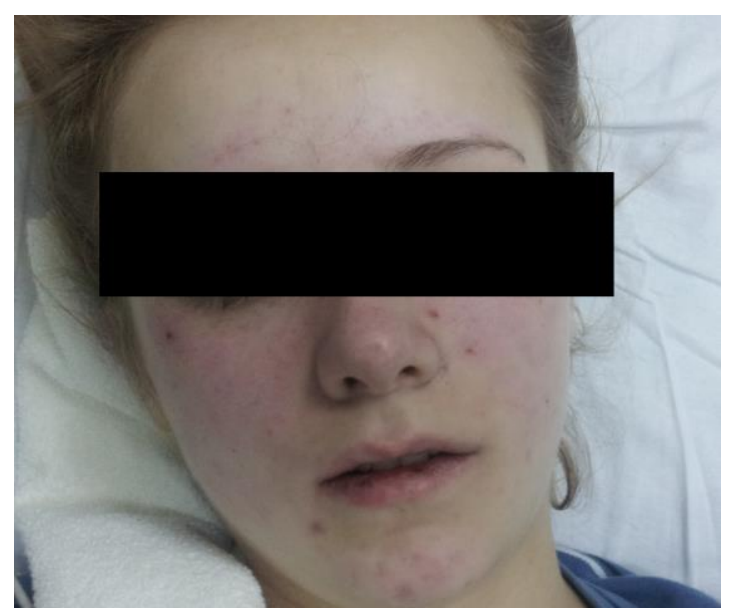

Fig. 3. "Butterfly" facial erythema

The neurologic examination revealed flaccid quadriplegia, generalized hypotonia, absent osteotendinous reflexes, bilateral Babinski sign, swallowing disorders and speech problems.

We continued paraclinical investigations and we found normochromic normocytic anemia $(\mathrm{Hb}=10.6 \mathrm{~g} / \mathrm{dl})$, pancitopenia, mild increase of ESR $(36 \mathrm{~mm} / \mathrm{h})$ and fibrinogen (156 mg/dl), marked elevation of transaminases $(A L T=337 \mathrm{U} / \mathrm{l}, \mathrm{AST}=836 \mathrm{U} / \mathrm{l})$, 
cholestasis $(\mathrm{GGT}=406 \mathrm{u} / \mathrm{l}$; Total Bilirubin = $1.48 \mathrm{mg} / \mathrm{dl}$; Direct Bilirubin $=1.09 \mathrm{mg} / \mathrm{dl}$ ).

The immunologic examination showed a normal value of rheumatoid factor (129). We were waiting the results for c-ANCA, p-ANCA, anti-dsDNA antibody, total ANA, cryoglobulines; proteins electrophoresis.

The electrocardiogram yielded sinus bradycardia, 45 BPM and low QRS voltage. Chest X-ray showed diffuse accentuation of bronchovascular markings.

We evaluated the criteria for systemic lupus erythematosus and we had 4 criteria from 11 (Table 1): cutaneous modifications (with " butterfly" facial erythema, petechial eruption and trophic disorders at distal phalanges, erythematous plaque of thighs favorable evolution under corticotherapy from red purple to pale pink (Figure 4); ulcerative lesions of oral mucosae and pharynx - bloody crusts; blood analysis: pancitopenia with lymphopenia $\quad(1100 / \mathrm{mmc}) ; \quad$ encephalitic neurologic damage of CNS.

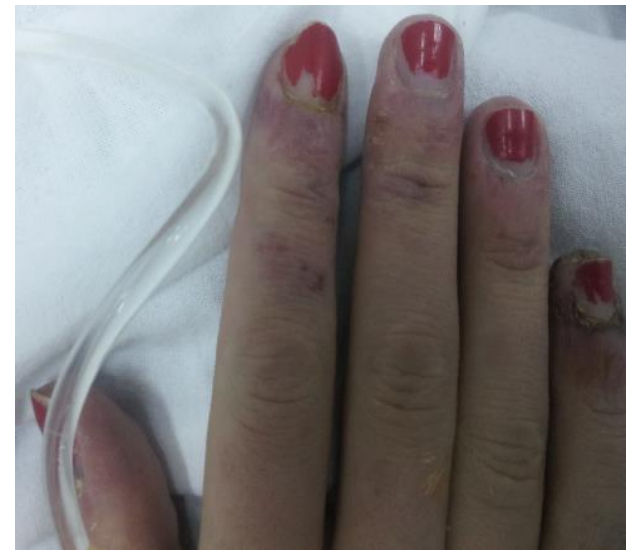

Fig. 4. Trophic disorders at distal phalanges, upper limbs

Table 1. ACR criteria for SLE (the diagnosis for SLE requires the presence of four or more of the following criteria simultaneously or in succession) [4] (a mnemonic to remember the eleven symptoms is "SOAP BRAIN MD")

\begin{tabular}{|c|c|}
\hline & Definition or examples \\
\hline Serositis & $\begin{array}{l}\text { Pleuritis - pleuritic pain, pleural rub, pleural effusion } \\
\text { Pericarditis - ECG changes, pericardial rub pericardial effusion }\end{array}$ \\
\hline Oral ulcers & Often painless sores \\
\hline Arthritis & Non erosive -2 or more peripheral joints affected \\
\hline Photosensitivity & Skin rash as result of unusual reaction to sun \\
\hline Blood & $\begin{array}{l}\text { Hematological disorder } \\
\text { Hemolytic anemia } \\
\text { Leucopenia } \\
\text { Lymphopenia } \\
\text { Trombocytopenia }\end{array}$ \\
\hline Renal disorder & $\begin{array}{l}\text { Proteinuria (with } 3+\text { or more protein noted in urinalysis specimen or } 0.5 \\
\text { g of protein/day) } \\
\text { Cellular casts in urine }\end{array}$ \\
\hline Antinuclear antibody & Antibodies to nucler constituents \\
\hline Immunological disorder & Anti-DNA antibodies, Anti-Sm antibodies, Antiphospholipid antibodies \\
\hline Neurological disorder & $\begin{array}{l}\text { Seizures } \\
\text { Psychosis }\end{array}$ \\
\hline Malar rash & Fixed erythema over the malar eminences \\
\hline Discoid rash & Erythematosus raised patches may scar \\
\hline
\end{tabular}

Upon receiving the results we noticed that the total ANAs were positive, the dsANA were $281.5 \mathrm{IU} / \mathrm{ml}$ (normal value $<20 \mathrm{IU} / \mathrm{ml}$ ), the complement was low C3c $<30 \mathrm{mg} / \mathrm{dl}$ (normal value is $90-180 \mathrm{mg} / \mathrm{dl}$ ), C4 $<6 \mathrm{mg} / \mathrm{dl}$ (normal value is $10-40 \mathrm{mg} / \mathrm{dl}$ ); circulating immune complexes $\mathrm{C} 1 \mathrm{q}$ was $36.26 \mathrm{RU} / \mathrm{ml}$ (normal value $<20$ ). 
Under corticosteroids (Methylprednisolone $750 \mathrm{ml} /$ day - 3 days; $500 \mathrm{ml}-1$ day) we obtained the regression from plegia to paresis and improvement of brainstem symptoms, but with worsening of pulmonary symptoms with the aspect of bronchopneumonia. It was necessary to do an endotracheal intubation and mechanical ventilation because of the oxygen desaturation (81\%) and polypnea (30/min).

The patient was transferred in Nephrology Clinic - ICU for plasmapheresis. After 2 sessions of plasmapheresis there was a worsening of neurologic symptoms (flaccid quadriplegia), respiratory failure, and bronchopneumonia with staphylococcus, gramnegative bacteria and Acinetobacter.

She was transferred back into the ICU of our hospital and we did a cerebral CT. We found hypodense areas mid-pons, bilateral lenticular in 1/2 anterior of caudate nucleus well bounded without pathological contrast; ischemic hypodense area on left external capsule with frontal extension.

Blood analysis in dynamics showed mild elevation of transaminases (ALT=96 IU//; AST=97 IU/I), normochromic normocytic anemia $(\mathrm{Hb}=7.1 \mathrm{~g} / \mathrm{dl})$, marked inflammatory syndrome with leukocytosis $19.000 /$ ul, increase of ESR $(28 \mathrm{~mm} / 1 \mathrm{~h})$ and fibrinogen $(243 \mathrm{mg} / \mathrm{dl})$.

We continued the treatment with antibiotics with 4th generation Cephalosporin and Meropenem according to DST. The rheumatologist recommended cyclophosphamide pulse therapy $(600 \mathrm{mg}$ i.v. $)$ and corticotherapy with Methylprednisolone i.v. 125 $\mathrm{mg} /$ day.

Despite this, the general state of the patient worsened and hyperthermia $42 \mathrm{gr} \mathrm{C}$ (106.7 gr F) appeared refractory to drugs and physical therapy. We considered that the fever was central with diencephalic implications. After a half hour she went into irreversible cardiorespiratory arrest.

The postmortem diagnosis was: necrotizing encephalitis of basal ganglia and medulla, brainstem syndrome, flaccid quadriplegia, acute respiratory failure, SLE, bronchopneumonia with staphylococcus and gram-negative bacteria, ascites, severe anemia, chronic autoimmune hepatitis.

\section{Discussions}

Severe neurological involvement in systemic lupus erythematosus is one of the most dreadful complications of the disease [5].

The American College of Rheumatology recognizes 19 SLE neuropsychiatric syndromes including psychosis, stroke, seizures and subtle abnormalities of cognitive function [6].

Neuro-psychiatric SLE should be considered as a possible differential diagnosis for rapid onset of neuro-psychiatric symptoms even in previously healthy individuals. SLE related central nervous system involvement is associated with a poor prognosis [7].

According to the ACR criteria for the diagnosis of SLE, our patient met 4 out of 11 characteristics (neurologic involvement, hematologic disorder, cutaneous lesions, oral ulcers) when diagnosed. Because of the rapid progression of the disease, we had to diagnose her without immunological results. Later, it was confirmed by ANA titer and antibodies to double-stranded DNA.

We suppose that the permeability across the blood brain barrier was increased by an infectious disease (acute pharyngitis) in the presented case and triggered the remarkable immunologic response. The problem remains what is the molecular support of such an important cerebral event.

\section{Conclusions}

Treatment of cerebral CNS continues to represent a major therapeutic challenge for clinicians in daily practice. The ideal drugs, doses and length of treatment are not yet well defined [5].

Endothelium is apparently a target for several pathogenic mechanisms that can account for the CNS manifestations of SLE.

The autoimmune response in SLE might affect endothelial cells through several mechanisms and different mediators: endothelial activation/damage may be mediated by $\mathrm{C} 1 \mathrm{q}$ - fixing immune-complexes through the involvement of the $\mathrm{C} 1 \mathrm{q}$ receptor expressed on the endothelial membrane. 
Moreover, C5a can induce heparansulphate release from endothelial cell membranes so promoting a procoagulant activity and the membrane attack complex might mediate von Willebrand secretion and tissue factor expression.

Circulating antibodies against constitutive or adhered endothelial cell molecules have been detected in SLE and shown to be able to induce an endothelial activation. The interaction between endothelial CD 40 and its specific ligand up regulates the adhesion

Authors declare no conflicts of interest.

\section{References}

1. Frieri M. Mechanisms of disease for the clinician: systemic lupus erythematosus. Ann Allergy Asthma Immunol 2013; 110(4):228-32 doi: 10.1016/j.anai.2012.12.010

2. The American College of Rheumatology nomenclature and case definitions for neuropsychiatric lupus syndromes. Arthritis Rheum 1999; 42(4):599-608. 10.1002/1529 0131(199904) 42:4<599:AID-ANR2>3.0.CO;2-F

3. Stock AD, Wen J, Putterman C. Neuropsychiatric Lupus, the Blood Brain Barrier, and the TWEAK/Fn14 Pathway. Front Immunol 2013; 4;484 doi: 10.3389/fimmu.2013. 00484.

4. Hochberg MC. Updating the American College of Rheumatology revised criteria for the classification of systemic lupus erythematosus. Arthritis Rheum 1997; 40:1725. molecule expression and the secretion of interleukin IL1 by endothelium. Proinflammatory cytokines and growth factors secreted by adjacent cells or by activated endothelial cells might act on the endothelium itself in a paracrine and autocrine manner further enhancing cell activation [8].

In conclusion, starting from our case of fulminant CNS lupus in a young woman and the absence of the response to conventional therapies we support the idea of research of molecular targets.

5. Barile-Fabris L, Ariza-Andraca R, Olguin-Ortega $L$, et al. Controlled clinical trial of IV cyclophosphamide versus IV methylprednisolone in severe neurological manifestations in systemic lupus erythematosus. Ann Rheum Dis 2005; 64(4):620-5.

6. Everett CM, Graves TD, Lad S, et al. Aggressive CNS lupus vasculitis in the absence of systemic disease activity. Rheumatology 2008; 47(1):107109 doi:10.1093/rheumatology/kem264

7. Rizos T, Siegelin M, Hähnel S, StorchHagenlocher B, Hug A. Fulminant onset of cerebral immunocomplex vasculitis as first manifestation of neuropsychiatric systemic lupus erythematosus (NPSLE), Lupus 2009; 18:361363;

8. Meroni PL, Tincani A, Sepp $N$, et al. Endothelium and the brain in CNS lupus. Lupus 2003; 12(12):919-28; DOI: 10.1191/0961203303 lu503oa. 